\title{
Review \\ Endocrine disrupting chemicals (phenol and phthalates) in the South African environment: a need for more monitoring
}

\author{
OO Olujimi ${ }^{1 *}$, OS Fatoki ${ }^{1}$, JP Odendaal ${ }^{1}$ and JO Okonkwo ${ }^{2}$ \\ ${ }^{1}$ Faculty of Applied Sciences, Cape Peninsula University of Technology, PO Box 652, Cape Town 8000, South Africa \\ ${ }^{2}$ Faculty of Natural Sciences, Tshwane University of Technology, Pretoria, South Africa
}

\begin{abstract}
There has been increasing concern about the impacts of exposure to chemical compounds with endocrine disrupting activities in the environment, especially aquatic environments, to wildlife and humans. South Africa is known to have used and abused most chemicals listed by developed and developing countries as endocrine-disrupting chemicals. Endocrinedisrupting chemicals have been reported in water, sediment and serum, as well as in fish tissue samples, at a level that could trigger endocrine disruption in humans and wildlife. Although some monitoring has been reported, particularly in water systems within the country, information on EDCs in other environmental matrices is scanty. The water systems monitored so far are very few. The strongest economy in Africa, and an emerging world economy depending on agriculture, mining, manufacturing and industry, needs to focus more on monitoring and to strengthen government organs and institutions to monitor and ensure environmental safety.
\end{abstract}

Keywords: Endocrine disrupting chemicals (EDCs), phenols, phthalates, monitoring, South Africa

\section{Introduction}

The endocrine system, also called the hormonal system, is one of the main systems for communicating, controlling and coordinating the body's activities in mammals (Ying et al., 2004). It works with the nervous system to regulate essential body functions. These body functions include energy metabolism, reproduction, growth and development, osmoregulation and homeostasis. The endocrine system also regulates reproductive processes and skeletal development (Bornman et al., 2007; Burger and Moolman, 2006; Ying et al., 2004; Vogel, 2004).

Endocrine disrupting chemicals (EDCs) consist of many natural and synthetic organic compounds, but are mostly manmade products such as alkylphenols, alkylphenols-ethonylates, and polychlorinated biphenyls (PCBs). Others include polychlorinated dibenzo-p-dioxins, polychlorinated dibenzofurans, organochlor pesticides, dichlorodiphenyl, dichloroethylene, nonylphenols, steroid hormones and phthalates (Arditsoglou and Voutsa, 2008; Hjelmborg et al., 2006; Mauricio et al., 2006). EDCs are able to cause abnormalities in invertebrate, fish, avian, reptilian, and mammalian species (Arditsoglou and Voutsa, 2008; Ferraz et al., 2007; Hjelmborg et al., 2006; Mauricio et al., 2006; Moder et al., 2007; Peng et al., 2006).

There has been debate for and against the occurrence of EDCs in humans. However, there are increased incidences of abnormalities in human sexual and cognitive development in some societies with speculation that these are being caused by EDC exposure (Campbell et al., 2006; Falconer et al., 2006; Matthiessen, 2000; Suliman et al., 2006; Solomon and Schettler, 2000). These abnormalities include low sperm count

* To whom all correspondence should be addressed.

용 +27 (0)73 1791443 ; fax: +27 (0)21 4603905 ; e-mail: 209076151@cput.ac.za

Received 29 October 2009; accepted in revised form 3 September 2010. and decrease in sperm quality (Van Wyk et al., 2003), and immunological and neurological effects (Patandin et al., 1999).

Over the last 2 decades there has been increasing concern about the likely impacts of exposure to chemical compounds with endocrine-disrupting activity in the environment (Game et al., 2006; Hecker and Giesy, 2008; Mauricio et al., 2006; Moder et al., 2007; Segner, 2005; Shin et al., 2007; Sumpter, 2005; Xue and $\mathrm{Xu}, 2006$ ). Endocrine-disrupting effects of chemicals were first documented in the 1930s but research was only accelerated in the late 1970s and early 1980s along 2 initially isolated pathways, i.e. human health effects and wildlife biology (Matthiesen, 2000; Moder et al., 2007; Trenholm et al., 2006; Vogel, 2004). The endocrine system's glands, hormones and their respective functions are listed in Table 1; Table 2 shows some EDCs with their common utilisations, and the target hormones and animals affected.

Considering that many of these compounds can elicit estrogenic responses at very low concentrations (parts per billion to parts per trillion), there is need for concern as many of the phthalate esters and phenolic compounds have been found at measurable concentrations in wastewater, surface waters, sediments, groundwater, and even drinking water in many countries (Cai et al., 2003; Cortazar et al., 2005; Huang et al., 2008, Sha et al., 2007).

\section{Mechanisms of endocrine disruption}

Endocrine disruptors initiate their disruption activities using one or more of the following mechanisms:

- By binding to receptors and mimicking or antagonising the effects of the endocrine hormones (Barcelo and Kettrup, 2004; Burger and Moolman, 2006; Jiao and Cheng, 2008; Sumpter, 2005; Vogel, 2004)

- By affecting the concentration of hormones through the altering of their synthesis or metabolism of natural 


\begin{tabular}{|l|l|l|}
\hline \multicolumn{2}{|c|}{ Endocrine system's glands, $\begin{array}{c}\text { Table 1 } \\
\text { and hormones and their functions }\end{array}$} \\
\hline Gland & Hormones & Functions \\
\hline Hypothalamus & Releasing hormones & Stimulate pituitary activity \\
\hline Pituitary & Trophic (stimulating) hormones & Stimulate thyroid, adrenal, gonadal and pancreatic activity \\
\hline Thyroid & Thyroid & Regulate metabolism, growth and development \\
\hline Adrenal & $\begin{array}{l}\text { Corticosteroid hormones } \\
\text { Catecholamines }\end{array}$ & Regulate metabolism and behaviour \\
\hline Pancreas & Insulin and glucagon & Regulate blood sugar levels \\
\hline Gonads & $\begin{array}{l}\text { Sex steroid hormones (estrogens and } \\
\text { androgens) }\end{array}$ & $\begin{array}{l}\text { Regulate development and growth, reproduction, immunity, } \\
\text { onset of puberty and bahaviour }\end{array}$ \\
\hline
\end{tabular}

Adapted from Bornman et al., 2007

\begin{tabular}{|c|c|c|c|}
\hline \multicolumn{4}{|c|}{$\begin{array}{c}\text { Table } 2 \\
\text { Endocrine disrupting chemicals, hormone target and animals affected }\end{array}$} \\
\hline Compound & Common usage & Target hormone & Animals affected \\
\hline \multicolumn{4}{|l|}{ Industrial chemicals } \\
\hline Bisphenol $\mathrm{A}^{\mathrm{b}}$ & Plasticiser & Thyroid, cortisol & Mammals, birds, fish \\
\hline $\begin{array}{l}\text { PCBs, Dioxins, PCP, } \\
\text { PCDFs }\end{array}$ & $\begin{array}{l}\text { Flame retardants, unitended by- } \\
\text { products during incineration }\end{array}$ & Estrogens & Reptiles, amphibians \\
\hline \multicolumn{4}{|l|}{ Phthalates $^{a}$} \\
\hline BBP, DEHP, D-n-BP & Plasticiser & Estrogens & Mammals, birds, fish, reptiles, amphibians \\
\hline \multicolumn{4}{|l|}{ Alkylphenols ${ }^{b}$} \\
\hline p-Nonylphenol & Plasticiser & Estrogens & Mammals, birds, fish, reptiles, amphibians \\
\hline \multicolumn{4}{|c|}{ Organochlorine Pesticides ${ }^{b}$} \\
\hline $\begin{array}{l}\text { DDT, DDE, Chlordane } \\
\text { Dieldrin, Heptachlor, } \\
\text { Lindane, Endosulfan } \\
\text { Oxychlordane, etc. }\end{array}$ & Insecticides & $\begin{array}{l}\text { Estrogens and } \\
\text { androgens }\end{array}$ & Mammals, birds, fish, reptiles, amphibians \\
\hline \multicolumn{4}{|l|}{ Heavy metals ${ }^{b}$} \\
\hline Cadmium, mercury, lead & Batteries, paints & Adrenaline, estrogens & Mammals, birds, fish \\
\hline
\end{tabular}

${ }^{a}$ Hill et al., 2001

${ }^{b}$ Zala and Penn, 2004

hormones (Bradlow et al., 1995; Rice et al., 2003; Sumpter, 2005; Toppari et al., 1996; Ying et al., 2004)

- By interfering with the signal between the different components of the hypothalamus-pituitary-endocrine gland axes (Clotfelter et al., 2004; Dawson, 2000; Kitano et al., 2006)

- By modifying the number of hormone receptors in a cell (Lascombe et al., 2000; Rajapakse et al., 2001; Soto et al., 1995; Welch et al., 1969).

\section{Routes of exposure of EDCs to humans and animals}

Human beings can be exposed to endocrine-disrupting chemicals via water, air, soil or food, through ingestion (i.e. oral), inhalation, and dermal absorption (Rice et al., 2003; US EPA, 1992; WHO, 2003), as with every other environmental contaminant. The major route of exposure to EDCs for young infants and children is via the oral route by direct ingestion of the chemicals, breast milk, infant formula, cow's milk, contaminated media like water, food, surface and carpet dust, toys and medical devices (Huang et al., 2008; Rice et al., 2003; Sathyanarayana, 2008).

Animals are also exposed to EDCs in the air, water and in their food (McKinlay et al., 2008; Clotfelter et al., 2004). EDCs enter animal bodies through the skin, gill, and even via the mother in utero or in ovo. EDCs, because they are lipid-soluble, tend to accumulate in animal's fat tissues. This problem is further aggravated by the process of biomagnification, in which chemical concentrations increase at higher trophic levels (Huang et al., 2008; McKinlay et al., 2008). In aquatic birds, contaminant concentrations are often 100 times greater in body tissue than in the surrounding water. In the case of marine animals, significant bioaccumulation has been observed in several species (Alatriste-Mondrageon et al., 2003). Top predators are essential for maintaining the integrity of food webs; thus, biomagnification of EDCs can affect entire ecosystems by harming species at the highest trophic levels (Clotfelter et al., 2004). The most common routes of human and animal exposure to EDCs are depicted in Fig. 1.

Some of these chemicals used as pesticides remain as residues in fresh and processed food (Bornman et al., 2007; Vogel, 2004). The effects of EDCs on the human body differ substantially from poisoning or toxic exposure which could cause cancer, physiological birth defects, gene mutation, cell damage or acute health effects (Campbell et al., 2006; Sharpe and Skakkabaek, 1993; Vogel, 2004). The health effects of EDCs may be pervasive throughout the planet due to fast and universal transport of chemicals through the world's atmosphere and oceans. Endocrine disruption may take longer time spans, or act selectively during certain stages of development or only in later generations for the effects to manifest (Falconer et al., 2006; Juvancy et al., 2008; Vogel, 2004). 


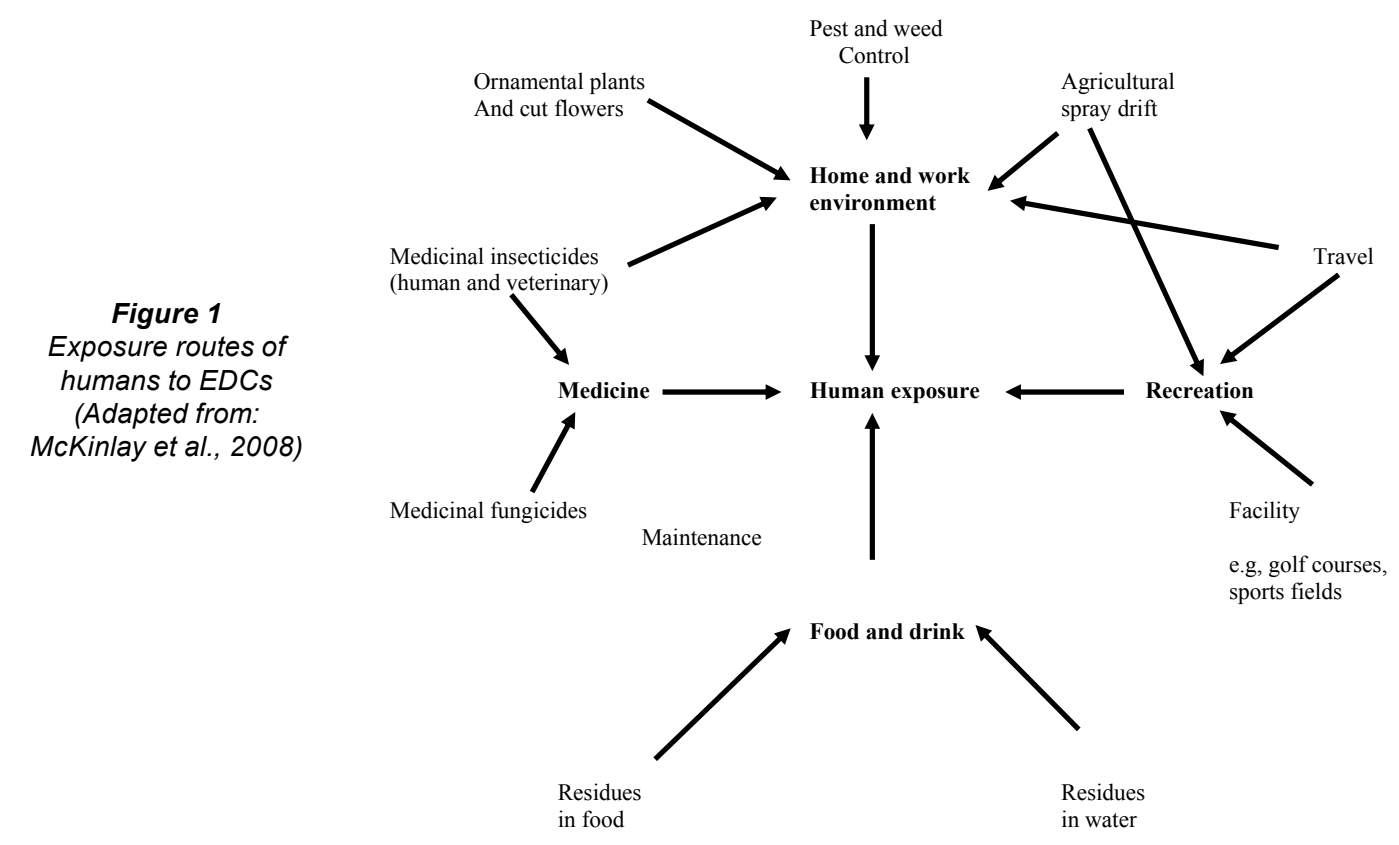

\section{Routes of exposure of EDCs to the aquatic environment}

There is growing concern regarding water quality; consequently industries around the world are faced with the challenge of ensuring a sustained and safe supply of drinking water from various sources. However, population growth, urbanisation, industrial development and associated changes in agricultural and land-use practices have contributed significantly to reducing water quality through naturally occurring and anthropogenic contamination (Falconer et al., 2006; Suliman et al., 2006).

Surface water contaminants include metals, carcinogens, synthetic chemicals, pharmaceuticals, veterinary and illicit drugs. Others are ingredients in cosmetics, personal care products, food supplements together with their respective metabolites and transformed products (Brasher and Wolff, 2004; Cortazar et al., 2005; Falconer et al., 2006). Some of these compounds and their metabolites are endocrinedisrupting chemicals and get into water through direct discharge of pharmaceuticals, chemicals, households, agricultural and industrial wastes. EDCs also get into water through accidental spills and indirect sources such as stormwater runoff (Falconer et al., 2006; Huang et al., 2008).

Drinking water sources can be contaminated due to flow of water through agricultural areas where agrochemicals are extensively used to improve crop yield (Falconer et al., 2006). EDCs are not only concentrated in the environment through biogeochemical processes but are also scavenged from water through sorption onto suspended materials, and deposited to be part of the bottom substrate. Aquatic wildlife appears to be particularly at risk since the aquatic environment is usually a sink for many hormonally-active chemicals, including industrial chemicals, pesticides, organochlorides, pharmaceuticals, natural and synthetic estrogens or phytoestrogens (Alatriste-Mondragon et al., 2003; Li et al., 2006; Luks-Betlej et al., 2001; Segner, 2005; Suliman et al., 2006; Xue and $\mathrm{Xu}, 2006$; Yuan et al., 2002).

Many aquatic ecosystems are faced with spatially or temporally alarming levels, and persistent complex mixtures, of EDCs, as a result from pollution with industrial chemicals (Schmidt et al., 2005). The aquatic environment is susceptible to pollution partly because there is very considerable intentional release of chemicals into rivers, lakes and the sea and partly because it receives a lot of accidental releases of chemicals through spills, runoff, wastewater from waste treatment plants and atmospheric deposition (Luks-Betley et al., 2001; Sanchez-Avila et al., 2009; Sumpter, 2005).

The main sources of endocrine-disrupting chemicals in rivers and lakes of Europe and North America are sewage effluents and agricultural chemical runoff. In developing countries, such as those in Africa and Asia, uncontrolled domestic and industrial discharge to waterways contributes tremendously to high levels of EDCs in aquatic environments (Falconer et al., 2006; Peng et al., 2006). The pressure of waste dumping or accidental spills has recently been on the increase with growing populations. The increasing use of water by people has contributed to the spatial and temporally alarming levels and complex mixtures of these chemicals (Falconer et al., 2006; Sumpter, 2005; Schmidt et al., 2005).

EDCs are introduced into the environment as byproducts of various technological processes. In the aquatic environment, the mobility of EDCs is increased when associated with fulvic or humic acids and particulates, which are often deposited in sediments, which usually determines their rates of transformation (Huang et al., 2008; Sun et al., 2006). They are transported through the food chain via benthic algae and invertebrates, which can be eaten by fish or birds. They may also undergo a series of processes such as biodegradation, dilution and photolysis once in waterways; activities that contribute to their elimination from the environmental water (Brasher and Wolff, 2004; Cortazar et al., 2005; Czaplicka, 2001; Petrovic et al., 2001). However, this creates potential routes of exposure of endocrine-disrupting chemicals to terrestrial and aquatic wildlife.

Phthalates, phenols and phenol derivatives have been included in the list of priority pollutants by the US Environmental Protection Agency (EPA) and the European Union (Llompart et al., 2002) due to their activities on aquatic and terrestrial animals. 
<smiles>COC(=O)c1ccccc1C(=O)OC</smiles>

Dimethyl phthalate (DMP)<smiles>CCCCOC(=O)c1ccccc1C(=O)OCCCC</smiles>

Dibutyl phthalate<smiles>CCOC(=O)c1ccccc1C(=O)OCC</smiles>

Diethyl phthalate (DEP)<smiles>CCCCC(CC)COC(=O)c1ccccc1C(=O)OCC(CC)CCCC</smiles>

Di-(2-ethylhexyl) phthalate (DEHP)<smiles>CCCCOC(=O)c1ccccc1C(=O)OCc1ccccc1</smiles>

Butyl benzyl phthalate (BBP)

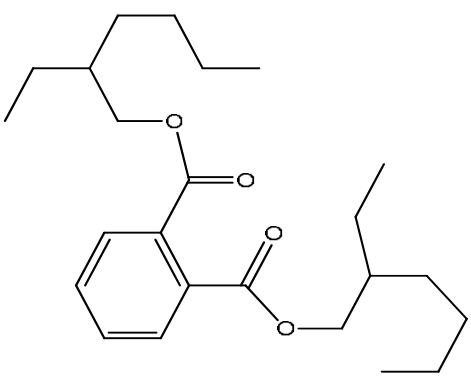

Dioctyl phthalate

Figure 2

Chemical structures of phthalates

\section{Phthalates}

\section{Background information on phthalic acid esters (PAEs)}

Phthalates or phthalic acid esters (PAEs) are diakyl or alkyl esters of 1,2 benzene carboxylic acid (Adeniyi et al., 2008; Alatriste-Mondragon et al., 2003; Luks-Betlej et al., 2001). They are formed when methanol, ethanol or other alcohols react with the carboxyl groups on the benzene ring of phthalic acids. The corresponding esters are formed with different alkyl chains, e.g., dimethyl phthalate (DMP), dibutyl phthalate (DBP) and di(2-ethylhexyl) phthalate (DEHP). PAEs are hydrophilic in nature and vary in structure (Fig. 2) and composition. Generally, the molecular weight of phthalates ranges between 194 and 396 with low melting points ranging from $-4.6^{\circ} \mathrm{C}$ to $5.5^{\circ} \mathrm{C}$ (Table 4).

\section{Application of phthalates}

PAEs have been widely used as plasticizers in the production of polyvinyl chloride (PVC) base plastics which include rubber, cellulose film, styrene, adhesives, coatings, pulp and paper manufacturing (Cortazar et al., 2005; Huang et al., 2008; Yuan et al., 2002; Rudel and Perovich, 2009). Other important usage of PAEs are in plumbing, pesticide formulations, non-ionic surfactants, construction materials, vinyl upholstery, table cloths, and shower curtains, to improve the mechanical properties of the plastic resin, particularly its flexibility and softness (Alatriste-Mondragon et al., 2003; Cortazar et al., 2005; Huang et al., 2008; Kayali et al., 2006; Ling et al., 2007; Luks-Betlej et al., 2001; Yuan et al., 2002; Rudel and Perovich 2009; Yuan et al., 2008)

The PAE component makes up 10 to $60 \%$ of plastic product to provide flexibility; the phthalate plasticizers are not chemically bonded to resin and therefore are easily released or leached during the life cycle of plastic products (Adeniyi et al., 2008; Yuan et al., 2008). Phthalates occur as components of plastics that are used for major domestic and industrial purposes. This includes teething rings, pacifiers, soft squeeze toys, plastic bottles, food containers and medical equipment. They are also parts of laboratory products (tubes, caps, gas chromatography septa, vinyl gloves), cosmetics and industrial solvents that are made from plastics (Adeniyi et al., 2008; Alatriste-Mondragon et al., 2003; De Jager et al., 1998; Huang et al., 2008; Kayali et al., 2006).

Globally, several millions of phthalate esters are produced and used annually as primary additives to polyvinyl chloride (PVC) based plastics (Adeniyi et al., 2008; AlatristeMondragon et al., 2003; Ling et al., 2007; Rudel and Perovich, 2009; Yuan et al., 2008). Phthalate esters migrate into environmental components during production and distribution processes, usage and disposal. Ways of entering the environment include aqueous leaching from plastics and waste, incineration of plastic waste, volatilization from resin matrices and wet deposition from the atmosphere (Muszkat et al., 1997; Ling et al., 2007; Luks-Betlej et al., 2001; Polo et al., 2005; Rudel and Perovich, 2009).

\section{Phthalates as EDCs}

Since PAEs are ubiquitous in our environment, the major sources of their exposure to man are shown in Table 3. Studies have revealed detectable levels of phthalate esters in samples of foodstuff, human mother's milk, dust, environmental samples (water, soil, sediment) and textiles with di (2-ethylhexyl) phthalate (DEHP) and di-n-butyl phthalate (DBP) recorded as the most abundant. Generally, phthalates are of low acute toxicity with short biologic half-lives 


\begin{tabular}{|l|l|}
\hline \multicolumn{2}{|c|}{ Possible sources of exposure to phthalates } \\
\hline $\begin{array}{l}\text { Phthalate parent } \\
\text { compound }\end{array}$ & Potential sources of exposure \\
\hline $\begin{array}{l}\text { Di-2-ethylhexyl } \\
\text { phthalate (DEHP) }\end{array}$ & $\begin{array}{l}\text { PVC-containing medical tubing, blood storage bags, medical devices, food contamination, food } \\
\text { packaging, indoor air, plastic toys, wall coverings, tablecloths, floor tiles, furniture upholstery, shower } \\
\text { curtains, garden hoses, swimming pool liners, rainwear, baby pants, dolls, some toys, shoes, automobile } \\
\text { upholstery and tops, packaging film and sheets, and sheathing for wire and cable }\end{array}$ \\
\hline $\begin{array}{l}\text { Diethyl phthalate } \\
\text { (DEP) }\end{array}$ & $\begin{array}{l}\text { Cosmetics, nail polish, deodorant, perfumes/cologne, lotions, aftershave, pharmaceuticals/herbal coating, } \\
\text { insecticide }\end{array}$ \\
\hline $\begin{array}{l}\text { Di-butyl phthalate } \\
\text { (DBP) }\end{array}$ & $\begin{array}{l}\text { Nail polish, make-up, aftershave, perfumes, pharmaceuticals/herbal coating, chemiluminescent glow } \\
\text { sticks }\end{array}$ \\
\hline $\begin{array}{l}\text { Di-n-octyl phthalate } \\
\text { (DnOP) }\end{array}$ & Children's toys \\
\hline $\begin{array}{l}\text { Butyl benzyl } \\
\text { phthalate (BBzP) }\end{array}$ & $\begin{array}{l}\text { Vinyl flooring, adhesives, sealants, food packaging, furniture upholstery, vinyl tiles, carpet tiles, and artifi- } \\
\text { cial leather; also used in certain adhesives }\end{array}$ \\
\hline $\begin{array}{l}\text { Di-methyl phthalate } \\
\text { (DMP) }\end{array}$ & Insecticides, indoor-air, adhesives, hairstyling products, shampoo, aftershave \\
\hline
\end{tabular}

Sources: Swan, 2008; Rudel and Perovich 2009

\begin{tabular}{|c|c|c|c|c|c|c|}
\hline \multicolumn{7}{|c|}{$\begin{array}{c}\text { Table } 4 \\
\text { Physical properties of selected PAEs }\end{array}$} \\
\hline & Dimethyl phthalate & Diethyl phthalate & $\begin{array}{l}\text { Dibutyl } \\
\text { phthalate }\end{array}$ & $\begin{array}{l}\text { Butylbenzyl } \\
\text { phthalate }\end{array}$ & \begin{tabular}{|l|} 
Di-n- \\
octylphthalate
\end{tabular} & $\begin{array}{l}\text { Di-(2- } \\
\text { ethylhexyl) } \\
\text { phthalate }\end{array}$ \\
\hline Abbreviation & DMP & DEP & DBP & BBP & DNOP & DEHP \\
\hline CAS RN & $131-11-3$ & $84-66-2^{\text {a }}$ & $84-74-2$ & $85-68-7^{\text {a }}$ & $117-84-0^{\text {a }}$ & $117-81-7$ \\
\hline Molecular weight & 194.1886 & $222.24^{\mathrm{a}}$ & 278.3474 & $312.36^{\mathrm{a}}$ & $390.6^{\mathrm{b}}$ & 390.5618 \\
\hline Molecular formula & $\mathrm{C}_{10} \mathrm{H}_{10} \mathrm{O}_{4}$ & $\mathrm{C}_{12} \mathrm{H}_{14} \mathrm{O}_{4}$ & $\mathrm{C}_{16} \mathrm{H}_{22} \mathrm{O}_{4}$ & $\mathrm{C}_{19} \mathrm{H}_{22} \mathrm{O}_{4}$ & $\mathrm{C}_{24} \mathrm{H}_{38} \mathrm{O}_{4}$ & $\mathrm{C}_{24} \mathrm{H}_{38} \mathrm{O}_{4}$ \\
\hline Density & 1.19 & $1.12^{\mathrm{a}}$ & 1.043 & $1.0^{\mathrm{a}}$ & & 0.9732 \\
\hline Melting point & $5.5^{\circ} \mathrm{C}^{\mathrm{b}}$ & $-40.5^{\circ} \mathrm{C}^{\mathrm{b}}$ & $-35^{\circ} \mathrm{C}$ & $61.3^{\circ} \mathrm{C}^{\mathrm{a}}$ & $-46^{\circ} \mathrm{C}^{\mathrm{b}}$ & $-50^{\circ} \mathrm{C}$ \\
\hline Boiling point & $283.7^{\circ} \mathrm{C}$ & $295^{\circ} \mathrm{C}^{\mathrm{a}}$ & $340^{\circ} \mathrm{C}$ & $92.5^{\circ} \mathrm{C}^{\mathrm{a}}$ & $390^{\mathrm{b}}$ & $386.9^{\circ} \mathrm{C}$ \\
\hline Water solubility & $4200 \mathrm{mg} \cdot \ell^{-1}$ at $20^{\circ} \mathrm{C}$ & $1100 \mathrm{mg} \cdot \ell^{-1}$ at $25^{\circ} \mathrm{C}$ & $11.2 \mathrm{mg} \cdot \ell^{-1 \mathrm{~b}}$ & $2.7 \mathrm{mg} \cdot \ell^{-1 \mathrm{~b}}$ & $0.0005 \mathrm{mg} \cdot \ell^{-1 \mathrm{~b}}$ & $0.003 \mathrm{mg} \cdot \ell^{-1 \mathrm{~b}}$ \\
\hline Physical state & $\begin{array}{l}\text { Colourless, oily } \\
\text { liquid with a slight } \\
\text { ester odour }\end{array}$ & $\begin{array}{l}\text { Colourless, oily } \\
\text { liquid }\end{array}$ & $\begin{array}{l}\text { Colourless, } \\
\text { oily liquid with } \\
\text { a very weak } \\
\text { aromatic odour }\end{array}$ & & & $\begin{array}{l}\text { Colourless, } \\
\text { oily liquid } \\
\text { with almost } \\
\text { no odour }\end{array}$ \\
\hline
\end{tabular}

${ }^{a}$ Luks-Betlej et al., 2001

${ }^{b}$ Huang et al., 2008

of approximately 6-12 hours (Duty et al., 2005). They are metabolised and eliminated within $48 \mathrm{~h}$ of exposure in vertebrates and are therefore not highly bioaccumulative in the system (van den Berg et al., 2003). However, the frequent use of phthalate-containing personal care and consumer products, along with the frequent detection of metabolites in random population samples, suggests that phthalate exposure is continuous and ubiquitous. Consequently, the endocrine-disrupting nature of some phthalates is potentially responsible for adverse effects on human reproduction and development.

Based on previous human and animal studies, phthalates have been classified according to their impact strength. DEHP has been included in Class B2 and has been shown to be embryotoxic and teratogenic (Altriste-Mondragon et al., 2003; Kayali et al., 2006; Latini et al., 2009), while butyl-benzyl phthalate (BBP) is in Class C (possible human carcinogen) and di-n-butyl phthalate (DBP), di-ethyl phthalate (DEP) and dimethyl phthalate (DMP) were included in Class D (not yet classified as human carcinogens (AltristeMondragon et al., 2003).

\section{Phenols}

\section{Background information on phenol}

Phenol and its derivatives are aromatic molecules containing hydroxyl $\left(\mathrm{OH}^{-}\right)$, methyl $\left(\mathrm{CH}_{3}\right)$, amide $\left(\mathrm{NH}_{2}\right)$ or sulphonic group (McNeely et al., 1979) attached to the benzene ring structure (Huang et al., 2007; Toniolo et al., 2007). Phenols are widely present in the environment and occur in nature as building blocks for plants (Baltussen et al., 1999; Santana et al., 2009; Santana et al., 2005). They are formed naturally from decomposition of leaves and wood as well as through human activity like water purification processes (Santana et al., 2009; Santana et al., 2005; Schmidt-Baumler et al., 1999).

Phenol and its methyl derivatives show a stronger tendency to adsorb onto solid matrices and some have been found to be toxic to fish and other aquatic life,. The compounds at very low concentration have adverse effects on taste and odour of water and fish (Baldwin and Debowski, 1988; Czaplicka, 2001; Schmidt-Baumler et al., 1999). The compounds are introduced into rivers, groundwater and soil directly through industrial 
<smiles>Oc1ccccc1</smiles>

Phenol (PH)<smiles>Oc1ccccc1Cl</smiles>

2-Chlorophenol (2-CP)<smiles>Oc1ccc(Cl)cc1Cl</smiles>

2,4-Dichlorophenol<smiles>O=[N+]([O-])c1ccccc1O</smiles>

2-Nitrophenol (2NP)<smiles>Oc1c(Cl)c(Cl)c(Cl)c(Cl)c1Cl</smiles><smiles>O=[N+]([O-])c1ccc([O-])c([N+](=O)[O-])c1</smiles><smiles>Oc1c(Cl)cc(Cl)cc1Cl</smiles><smiles>Oc1ccc(Nc2ccccc2)cc1</smiles>

Pentachlorophenol (PCP) 2,4-Dinitrophenol (2,4-DNP)

2,4,6-Trichlorophenol (TCP) 4-Nitrophenol (4NP)<smiles>Cc1cc([N+](=O)[O-])cc(C#N)c1O</smiles>

4,6-Dinitroorthocresol $(4,6-\mathrm{DNOC})$<smiles>Cc1ccc(O)c(C)c1</smiles>

2,4-Dimethylphenol $(2,4-\mathrm{DMP})$<smiles>Cc1cc(O)ccc1Cl</smiles>

(4-C 3MP)

Figure 3

Chemical structures of some phenolic compounds classified as priority pollutants

effluents or indirectly through natural or synthetic chemicals (Bagheri et al., 2004; Ribeiro et al., 2002; Suliman et al., 2006). They are considered major environmental risks, with speculations of suspected endocrine disruptor or carcinogen effects (Bagheri et al., 2004; Suliman et al., 2006).

\section{Application of phenol}

Industrially, phenols and their derivatives are used in several useful products. They are used to manufacture chemicals such as pesticides, explosives, dyes, for pulp bleaching with chlorine, in wood preservatives, insecticides, herbicides, antioxidants, adhesives, plastics and synthetic intermediates (Baldwin and Debowski, 1988; Cledera-Castro et al., 2006; Czaplicka, 2001; Hartung et al., 2007; Ozkaya, 2005; Ribeiro et al., 2002; Santana et al., 2009; Santana et al., 2005; Suliman et al., 2006).

Globally, the production of phenolic compounds is over 300000 tons per annum. They have been widely used in the last 40 years as components of detergents, emulsifiers, dispersants and anti-foamers (Gabriel et al., 2007; Petrovic et al.,

\begin{tabular}{|c|c|c|c|c|c|c|c|}
\hline \multicolumn{8}{|c|}{$\begin{array}{c}\text { Table } 5 \\
\text { Physical properties of selected phenolic compounds }\end{array}$} \\
\hline & $\begin{array}{l}\text { Molecular } \\
\text { formula }\end{array}$ & $\begin{array}{l}\text { Molecular } \\
\text { formula }\end{array}$ & $\begin{array}{l}\text { Boiling } \\
\text { point }\left({ }^{\circ} \mathrm{C}\right)\end{array}$ & $\begin{array}{l}\text { Melting } \\
\text { point }\left({ }^{\circ} \mathrm{C}\right)\end{array}$ & pKa & $\begin{array}{c}\text { Log Kow } \\
\text { (Octanol/ } \\
\text { water) }\end{array}$ & $\begin{array}{c}\text { Water } \\
\text { solubility } \\
\text { gl-1 at } 20^{\circ} \mathrm{C}\end{array}$ \\
\hline Phenol & $\mathrm{C}_{6} \mathrm{H}_{5} \mathrm{OH}^{\mathrm{b}}$ & $94.11^{\mathrm{a}}$ & $181.7^{\mathrm{c}}$ & $40.5^{\mathrm{c}}$ & $10.0^{\mathrm{b}}$ & $1.46^{\mathrm{a}}$ & $83^{\mathrm{b}}$ \\
\hline 2-Methyl 4,6-dinitrophenol & $\mathrm{C}_{8} \mathrm{H}_{10} \mathrm{O}^{\mathrm{d}}$ & $122.17^{\mathrm{i}}$ & $211^{\mathrm{c}}$ & $22-23$ & $10.58^{\mathrm{d}}$ & $2.30^{\mathrm{a}}$ & $0.05^{\mathrm{c}}$ \\
\hline 4-Chloro-3-methyl phenol & $\mathrm{C}_{7} \mathrm{H}_{7} \mathrm{ClO}^{\mathrm{a}}$ & $142.58^{\mathrm{b}}$ & $235^{b}$ & $63-65^{c}$ & $9.6^{\mathrm{c}}$ & $3.10^{\mathrm{c}}$ & $4^{\mathrm{e}}$ \\
\hline 2-Chlorophenol & $\mathrm{C}_{6} \mathrm{H}_{5} \mathrm{ClO}$ & $128.6^{\mathrm{e}}$ & $175^{\mathrm{e}}$ & $9.3^{\mathrm{e}}$ & $8.56^{\mathrm{c}}$ & $2.15^{\mathrm{a}}$ & $28.5^{\mathrm{e}}$ \\
\hline 2,4-Dichlorophenol & $\mathrm{C}_{6} \mathrm{H}_{4} \mathrm{Cl}_{2} \mathrm{O}$ & $163^{\mathrm{e}}$ & $210^{\mathrm{e}}$ & $45^{\mathrm{e}}$ & $7.85^{\mathrm{b}}$ & $3.06^{\mathrm{a}}$ & $4.5^{\mathrm{b}}$ \\
\hline 2-Nitrophenol & $\mathrm{C}_{6} \mathrm{H}_{5} \mathrm{NO}_{3}$ & $139.1^{\mathrm{c}}$ & $216^{\mathrm{c}}$ & $45-46^{\mathrm{d}}$ & $7.23^{\mathrm{b}}$ & $1.89^{\mathrm{c}}$ & $2.5^{\mathrm{b}}$ \\
\hline 4-Nitrophenol & $\mathrm{C}_{6} \mathrm{H}_{5} \mathrm{NO}_{3}$ & $139.11^{\mathrm{d}}$ & $279^{\mathrm{d}}$ & $113-114^{\mathrm{e}}$ & $7.08^{\mathrm{c}}$ & $2.04^{\mathrm{c}}$ & $11.6^{\mathrm{e}}$ \\
\hline 2,4-Dinitrophenol & $\mathrm{C}_{6} \mathrm{H}_{4} \mathrm{~N}_{2} \mathrm{O}_{5}$ & 184.106 & $113^{\mathrm{d}}$ & 108 & $3.94^{\mathrm{c}}$ & $1.67^{\mathrm{c}}$ & 5.45 \\
\hline $2,4,6-\mathrm{TCP}$ & $\mathrm{C}_{6} \mathrm{H}_{3} \mathrm{Cl}_{3} \mathrm{O}^{\mathrm{e}}$ & $197.45^{\mathrm{c}}$ & $197.45^{\mathrm{c}}$ & $246^{\mathrm{c}}$ & $69^{c}$ & $6.15^{\mathrm{c}}$ & $3.69^{\mathrm{a}}$ \\
\hline Pentachlorophenol (PCP) & $\mathrm{C}_{6} \mathrm{HCl}_{5} \mathrm{O}^{\mathrm{b}}$ & $266.34^{\mathrm{b}}$ & $309-310^{\mathrm{b}}$ & $190-191^{\mathrm{e}}$ & $4.7^{\mathrm{b}}$ & $5.12^{\mathrm{a}}$ & $0.01^{\mathrm{a}}$ \\
\hline
\end{tabular}

${ }^{a}$ Montero et al., 2005

${ }^{c}$ Castillo et al., 1997

${ }^{b}$ Fiamegos et al., 2003

${ }^{e}$ Krijgsheid and Van der Gen, 1986

${ }^{d}$ Cledera-Castro et al., 2005 
2002; Schmidt-Baumler et al., 1999). Phenol is also used in the pharmaceutical industry for the production of aspirin (Suliman et al., 2006). Sixty percent of phenolic compounds that are introduced into sewage are released into the environment with $85 \%$ being in the form of potentially estrogenic degradation products (Petrovic et al., 2002). Phenolic derivatives are among the most important pollutants that are widely present in the environment. These compounds are used in several industrial processes.

\section{Methods for detection and monitoring of EDCs}

Established analytical methods are available for many of the compounds designated as EDCs. Most developed countries like the US and the members of the EU have established regulatory authorities and requirements for chemical and biological analytical procedures for testing pesticides, metals, industrial chemicals and PCBs in food and environmental matrices. Despite the institutional frameworks for the analysis of the designated compounds (hormones, drugs and personal care products), suitable instrumental techniques or standard methods of analysis are lacking. Different methods used to assess the occurrence, distribution and characterisation of phthalates and phenolic compounds in the environmental matrices and human samples in different countries around the world are summarised in Table 6.

\section{Extraction of EDCs in liquid samples}

Extraction of EDCs from liquid samples is based on liquidliquid extraction (LLE) which is often followed by column chromatography cleanup and gas chromatography (GC) or high performance liquid chromatography (HPLC) determination using array of detectors (Cai et al., 2003; Ling et al., 2007; Zhou et al., 2005). LLE is a well developed, efficient and precise method; it requires time and consumption of large volumes of organic solvents (Santana et al., 2009; Santana et al., 2005; Zhou et al., 2005). EDCs often vary in concentration with different ecological risks in different environmental compartments. LLE has remained the preferred extraction

\begin{tabular}{|c|c|c|c|c|c|}
\hline \multicolumn{6}{|c|}{$\begin{array}{c}\text { Table } 6 \\
\text { Matrix, methods and concentration of endocrine disrupting chemicals (phenols and phthalate esters) }\end{array}$} \\
\hline Country & $\begin{array}{l}\text { Environmental } \\
\text { matrix }\end{array}$ & Method & $\begin{array}{l}\text { Concentration/ Detection } \\
\text { range }\end{array}$ & Compound & Reference \\
\hline China & Water & GC-FID & $0.05-3.91 \mu \mathrm{g} \cdot \ell^{-1}$ & phthalate esters & Li et al., 2006 \\
\hline Taiwan & Water & GC-MS/ GC-ECD & $50-100 \mu \mathrm{g} \cdot \ell^{-1}$ & phthalate esters & Yuan et al., 2002 \\
\hline China & Water & HPLC-UV & $3.1-5.8 \mu \mathrm{g} \cdot \ell^{-1}$ & phthalate esters & Cai et al., 2003 \\
\hline South Africa & Water & GC-FID & $0.03-2309 \mu \mathrm{g} \cdot \ell^{-1}$ & phthalate esters & Fatoki and Noma, 2002 \\
\hline $\begin{array}{l}\text { Poland and } \\
\text { Germany }\end{array}$ & Water & GC-MS & $0.015-0.06 \mu \mathrm{g} \cdot \mathrm{m}^{-1}$ & phthalate esters & Luks-Betlej et al., 2001 \\
\hline Spain & Water & SPME/HPLC & $9-22 \mu \mathrm{g} \cdot \ell^{-1}$ & phthalate esters & Kayali et al., 2006 \\
\hline China & Water & HPLC-UV & $1.0-3.8 \mu \mathrm{g} \cdot \ell^{-1}$ & phthalate esters & Ling et al., 2007 \\
\hline Spain & Water & \begin{tabular}{|l|} 
DSPME/ \\
HS-SPME/GC-MS
\end{tabular} & $<$ LOD to $6172 \mathrm{pg} \cdot \mathrm{m} \ell^{-1}$ & phthalate esters & Polo et al., 2005 \\
\hline China & $\begin{array}{l}\text { Water } \\
\text { Sediment } \\
\text { Particulate matter } \\
\end{array}$ & \begin{tabular}{|l|} 
GC-FID \\
GC-FID \\
GC-FID \\
\end{tabular} & $\begin{array}{l}3.99 \times 10^{-3}-45.45 \times 10^{-3} \mathrm{mg} \cdot \mathrm{kg}^{-1} \\
40.56-94.22 \mathrm{mg} \cdot \mathrm{kg}^{-1} \\
30.52-63.96 \mathrm{mg} \cdot \mathrm{kg}^{-1}\end{array}$ & $\begin{array}{l}\text { phthalate esters } \\
\text { phthalate esters } \\
\text { phthalate esters }\end{array}$ & $\begin{array}{l}\text { Sha et al., } 2007 \\
\text { Sha et al., } 2007 \\
\text { Sha et al., } 2007 \\
\end{array}$ \\
\hline South Africa & Soil & GC-FID & $3.8-48.89 \mathrm{ng} \cdot \mathrm{kg}^{-1}$ & phthalate esters & Adeniyi et al., 2008 \\
\hline Italy & Breast milk & $\mathrm{LC} / \mathrm{LC}-\mathrm{MS} / \mathrm{MS}$ & $8.4-18.8 \mu \mathrm{g} \cdot \ell^{-1}$ & phthalate esters & Latini et al., 2009 \\
\hline Taiwan & & GC-MS & $\begin{array}{l}0.05-46.5 \mathrm{mg} \cdot \mathrm{kg}^{-1} \\
1.4-253 \mathrm{mg} \cdot \mathrm{kg}^{-1} \\
\end{array}$ & $\begin{array}{l}\text { phthalate esters } \\
\text { phthalate esters }\end{array}$ & $\begin{array}{l}\text { Huang et al., } 2008 \\
\text { Huang et al., } 2008 \\
\end{array}$ \\
\hline Oman & $\begin{array}{l}\text { Water } \\
\text { Water }\end{array}$ & $\begin{array}{l}\text { RP-HPLC } \\
\text { GC-MS }\end{array}$ & $\begin{array}{l}0.1-0.9 \mu \mathrm{g} \cdot \ell^{-1} \\
5-22 \mathrm{ng} \cdot \ell^{-1}\end{array}$ & $\begin{array}{l}\text { Phenols } \\
\text { Phenol }\end{array}$ & $\begin{array}{l}\text { Suliman et al., 2006; } \\
\text { Bagheri et al., } 2007\end{array}$ \\
\hline Spain & $\begin{array}{l}\text { Water } \\
\text { Sediment }\end{array}$ & \begin{tabular}{|l|l} 
HPLC \\
GC-MS \\
\end{tabular} & $3-76 \mu \mathrm{g} \cdot \ell^{-1}$ & Phenol & $\begin{array}{l}\text { Cledra-castro et al., } 2006 \\
\text { Czaplicka, } 2001\end{array}$ \\
\hline Italy & Leachate & SPME/GC-MS & $0.005-2.4 \mu \mathrm{g} \cdot \ell^{-1}$ & Phenol & Ribeiro et al., 2002 \\
\hline China & Sediment & GC-MS & $0.6-664.5 \mathrm{ng} \cdot \mathrm{g}^{-1}$ & Phenol & Peng et al., 2006 \\
\hline China & Wastewater & GC-FID & $0.47-9.01 \mu \mathrm{g} \cdot \ell^{-1}$ & Phenol & Zhou et al., 2005 \\
\hline China & Water & GC-NCI-MS & $17-685 \mathrm{ng} \cdot \ell^{-1}$ & Phenol & Zhao et al., 2009 \\
\hline Japan & Soil & GC-MS & $1.0 \mathrm{ng} \cdot \mathrm{g}^{-1}$ & Phenol & Helaleh et al., 2001 \\
\hline China & Water & SPE/GC-FID & $0.02-0.701 \mu \mathrm{g} \cdot \ell^{-1}$ & Phenol & Wang et al., 2006 \\
\hline Germany & Urine & HPLC/MS/MS & $0.1-13.6 \mathrm{ng} \cdot \mathrm{kg}^{-1}$ b.w & Phthalate esters & Seckin et al., 2009 \\
\hline Sweden & $\begin{array}{l}\text { Breast milk } \\
\text { Blood } \\
\text { Urine } \\
\end{array}$ & $\begin{array}{l}\text { GC-MS } \\
\text { GC-MS } \\
\text { GC-MS } \\
\end{array}$ & $\begin{array}{l}0.06-305 \mathrm{ng} \cdot \mathrm{m}^{-1} \\
0.06-305 \mathrm{ng} \cdot \mathrm{m} \ell^{-1} \\
0.06-305 \mathrm{ng} \cdot \mathrm{m} \ell^{-1}\end{array}$ & $\begin{array}{l}\text { Phthalate esters } \\
\text { Phthalate esters } \\
\text { Phthalate esters } \\
\end{array}$ & \begin{tabular}{|l|} 
Hogberg et al., 2008 \\
Hogberg et al., 2008 \\
Hogberg et al., 2008 \\
\end{tabular} \\
\hline China & Soil & GC-MS & $\mathrm{ND}-293 \mu \mathrm{g} \cdot \mathrm{g}^{-1}$ & Phthalate esters & Zeng et al., 2009 \\
\hline South Africa & Water & GC-MS & ND-119 $\mu \mathrm{g} \cdot \ell^{-1}$ & $\begin{array}{l}\text { Phthalate } \\
\text { esters/p-NP }\end{array}$ & Mahomed et al., 2008 \\
\hline
\end{tabular}


method for liquid samples because it is cheap compared to other methods like solid phase extraction (SPE), solid phase micro-extraction (SPME) and microwave accelerated extraction (MAE) (Piug and Barcelo, 1996; Polo et al., 2005; Satana et al., 2005). However, use of large amounts of generally toxic and inflammable organic solvents, formation of emulsions and losses during cleanup are still trailing the use of LLE.

The procedure is thus becoming less popular with the introduction of the solid phase extraction (SPE) technique. SPE is currently used in sample preparation for determination of trace EDCs in environmental, drug and biological samples. It offers a faster and easier manipulation, higher concentration factors and requires smaller amounts of organic solvents (Eberlin and Cesar de Silva, 2008; Zhou et al., 2005). Disposable cartridges for SPE have been in use for than 20 years. The desire to decrease the use of organic solvent, especially dichloromethane which is suspected to be carcinogenic, has encouraged the requirement for solvent-free procedures. This has greatly contributed to the rapid growth in the demand for this method at the expense of LLE. Selection of SPE cartridges with particular sorbent materials also plays a key role in the achievement of high and reproducible recovery of analytes in environmental samples. Most sorbents used in SPE are porous silica particles bounded with C-18 or other hydrophobic alkyl groups such as strene-divinylbenzene. Recovery of organic compounds by SPE is highly dependent on the polarity of the eluent (Patrolecco et al., 2004).

\section{Extraction of EDCs in solid samples}

Studies on the determination of EDCs in solid environmental matrices are scarce compared to those carried out on liquid samples. Samples such as sludge and sediments are very complex and can be subjected to various forms of interference during extraction and separation procedures. EDCs are usually present at very low concentrations in these samples. This therefore requires effective sample pre-treatment. Such pretreatment involves extraction and purification prior to analysis by GC or HPLC. Extraction of the compounds is often performed using soxhlet extraction, sonication with polar organic solvents or mixtures of them, and most recently by accelerated solvent soxhlet extraction (Diaz-Cruz et al., 2003; Noppe et al., 2007; Santana et al., 2009; Ternes et al., 2002).

However, prior to their extraction, solid samples, e.g., sludge, soil and sediments, are dried and homogenised by air-drying or lyophilisation (MacDowell and Metcalfe, 2001; Petrovic and Barcelo, 2000) or by mixing with baked sodium sulphate until a free-flowing powder is obtained. Often, solid samples are extracted with dichloromethane (Fatoki et al., 2010), different mixtures of hexane and other solvents (Petrovic et al., 2002) or ethyl acetate. Ethyl acetate is preferred to chlorinated solvent like DCM due to its suspected carcinogenic effect on humans and strong water-polluting properties.

\section{Gas chromatography-mass spectrometry (GC-MS) methods}

Phenols and phthalate esters have been determined in environmental matrices through the use of different analytical techniques, e.g., high-performance liquid chromatography (HPLC) with ultraviolet, fluorescence, electrochemical or mass spectrometric (MS) detection, as well as gas chromatography (GC) coupled with sensitive and specific detection systems (Huang et al., 2008; Liu et al., 2004; Zeng at al., 2009). GC-MS has been widely used in the determination, analysis and monitoring of phthalates and phenolic compounds (Liu et al., 2004; Psillakis et al., 2004; Rompa et al., 2003; Zhao et al., 2009). EDCs with hydroxyl groups are usually derivatized for $\mathrm{GC}$ analysis.

It is well known that the oxygen atom possessed by hydroxyl has a weak nuclephilicity owing to its low electron density. Therefore, the derivatization procedures for hydroxyl compounds need either high temperatures or longer reaction periods, which makes the derivatized compounds convenient to be analysed on GC with ECD or MS.

The high polarity of phenols affects their chromatographic resolution and usually results in broad and tailed peak heights (Heberer and Stan, 1997; Santana et al., 2009; Schummer et al., 2009; Rompa et al., 2003; Zhao et al., 2009). These problems are often overcome by derivatizing free phenols to less polar compounds, more volatile and thermally stable, and by giving larger and more selective analytical responses through the use of derivatising reagents (Helaleh et al., 2001; Santana et al., 2009; Schummer et al., 2009; Rompa et al., 2003; Zhao et al., 2009).

Phenol and other phenolic compounds are usually derivatized either before or after extraction, or with an on-column reagent in the GC-injector (Helaleh et al., 2001; Rompa et al, 2003). Derivatization reactions used in GC analysis of phenolic compounds are in 4 categories, i.e. acylation, alkylation, esterification, and silylation (Helaleh et al., 2001; Moghadam et al., 2008; Rompa et al, 2003). In derivatization reactions, methylation by diazomethane is slow and carcinogenic while esterification by acetic hydride does not sufficiently improve the separation and derivatization of mono-nitro compounds (Heberer and Stan, 1997). Silylation of all the derivatization has shown to improve chromatographic parameters such as accuracy, reproducibility, sensitivity and resolution. Other advantages include peak tailing suppression, enhancement of thermal stability and product ruggedness (Li and Park, 2001; Saraji and Bakhshi, 2005).

\section{Evidence and monitoring of EDCs (phenol and phthalates) in the South African environment}

South Africa has experienced rapid industrial and urban growth especially in the increase in number of small-scale industries (Mahomed et al., 2008). South Africa is known to have used and abused most chemicals listed by developed and developing countries as endocrine-disrupting chemicals (Burger and Nel, 2008). The presence of EDCs has been reported in the South African environment, which therefore exposes humans and wildlife to possible health problems (Aneck-Hahn et al., 2009; Burger and Moolman, 2006; Fatoki and Noma, 2002; Mahomed et al., 2008). Many water bodies in the country receive significant inputs of natural and synthetic chemicals (from both point- and diffuse sources) which often act as EDCs, thus constituting a threat to reproductive health of aquatic organisms (Burger and Moolman, 2006; Govender et al., 2007).

South Africa, as an emerging world economy depending largely on agriculture, chemical industries and mining, still faces challenges in the area of proper waste disposal from a number of industrial and agricultural sources (Aneck-Hahn et al., 2009; Bornman et al., 2007; Fatoki et al., 2002; Mahomed et al., 2008). An audit of wastewater in Pretoria (Mahomed et al., 2008) and water, sediment and serum samples (Bornman et al., 2007) confirmed the presence of EDCs. This weight of evidence gathered on EDCs in the South African environment has posed a huge challenge to water resource management. 
South Africa is faced with the problems of limited water resources with most rural and urbanised settlements depending on the available resources for their consumption, industrial and agricultural usage.

In addition, most research in South Africa on EDCs is centred on estrogenic compounds, such as 17- $\beta$ estradiol, $p$-Nonyl phenol, PCBs, estrone, estriol, organochlorine pesticides, and heavy metals (Aneck-Hahn et al., 2009; Barnhoorn et al., 2004; Mahomed et al., 2008; Van Wyk et al., 2003). It is evident from various studies that EDCs are capable of initiating various health disorders in aquatic organisms. Van Wyk et al. (2008) reported the testicular effects of some EDCs compound and metals on Clarias gariepinus. The impact of $17-\beta$ estradiol to stimulate vitellogenin (VTG) on Xenopus laevis has also been reported (Barnhoorn et al., 2004).

Though significant concentration of phthalates have been reported in some environmental samples in the country (Adeniyi et al., 2008; Fatoki and Noma, 2002; Fatoki et al., 2010), there has been paucity in research on phenols on the priority list until recently (Abboo and Pletschke, 2010; Baldwin and Debowski, 1988). Concentrations of some of the compounds have been reported in drinking water, lakes and swimming pools; inhibitory effects on $\beta$-D-galactosidase and $\beta$-Dglucuronidase have also been reported (Abboo and Pletschke, 2010). Assessing the potential ecological and health impacts of EDCs (phenol and phthalate esters) in the country's environment requires investigation of level and distribution in both aquatic and terrestrial systems.

Areas of significant importance that have been neglected in the monitoring of EDCs in the South African environment are treated wastewater from municipal treatment plants and landfill sites. Though wastewaters from households and industries are usually treated before they are released into in the river systems, studies have shown that they often contain EDCs. In addition, leachates from landfill sites are treated before onsite application, processes that can result in contamination of the water table by EDCs.

\section{Conclusion}

It is of importance to monitor terrestrial and aquatic environments close to agricultural, mining and chemical industries as wastes from these activities contribute immensely to the availability of EDCs in environmental compartments. To cover the vacuum created by the lack of research into these EDCs (phenols and phthalate esters) in the South African environment, future research will focus on the level, distribution, spatial and temporal availability, and possibly the risk assessment, of some of these compounds in South African environments.

\section{References}

ABBOO S and PLETSCHKE BI (2010) Effect of phenolic compounds on the rapid direct enzymatic detection of $\beta$-D-galactosidase and $\beta$-D-glucuronidase. Water $S A 36$ (1) 133-138

ADENIYI A, DAYOMI M, SIEBE P and OKEDEYI O (2008) An assessment of the levels of phthalate esters and metals in the Muledane open dump, Thohoyandou, Limpopo Province, South Africa. Chem. Cent. J. 29 DOI: 10.1186/1752-153X-2-9.

ALATRISTE-MONDRAGON F, IRANPOURB R and AHRINGA BK (2003) Toxicity of di-(2-ethylhexyl) phthalate on the anaerobic digestion of wastewater sludge. Water Res. 37 1260-1269.

ANECK-HAHN NH, BORNMAN MS and DE JAGER C (2009) Oestrogenic activity in drinking waters from rural area in the Waterberg District, Limpopo Province, South Africa. Water SA 35 (3) $245-252$
ARDITSOGLOU A and VOUTSA D (2008) Determination of phenolic and steroid endocrine disrupting compounds in environmental matrices. Environ. Sci. Pollut. Res. 15 (3) 228-236

BALDWIN DA and DEBOWSKI JK (1988) Determination of phenols by HPLC to PPT levels. Chromatographia 26 186-190.

BALTUSSEN E, DAVID F, SANDRA P, JANSSEN H and CRAMERS C (1999) Automotive sorptive extraction thermal desorption-gas chromatography mass spectrometry analysis: Determination of phenols in water samples. J. Microcolumn Sep. 11 (6) 471-474.

BAGHERI H, SABER A and MOUSAVI SR (2004) Immersed solvent microextraction of phenol and chlorophenols from water samples followed by gas chromatography-mass spectrometry. J. Chromatogr. 1046 27-33.

BARCELO D and KETTRUP A (2004) Endocrine disruptors. Anal. Bioanal. Chem. 378 547-548.

BORNMAN R, VAN VUREN, J, BOUWMAN H, DE JAGER T, GENTHE B and BARNHOORN I (2007) Endocrine Disruptive Activity and the Potential Health Risk in the Rietvlei Nature Reserve. WRC Report No. 1505/1/07. Water Research Commission, Pretoria.

BARNHOORN IEJ, BORNMAN MS, PIETERSE GM and VAN VUREN JHJ (2004) Histological evidence of intersex in feral sharptooth catfish (Clarias gariepinus) from an estrogen-polluted water source in Gauteng, South Africa. Environ. Toxicol. 19 (6) 603-608. DOI: 10.1002/tox.20068.

BRADLOW HL, DAVIS DL, LIN G, SEPKOVIC D and TIWARI R (1995) Effects of pesticides on the ratio of 16alpha/2-hydroxyestrone: A biological marker of breast cancer risk. Environ. Health Perspect. 103 143-150.

BRASHER AMD and WOLFF RH (2004) Relations between land use and organochlorine pesticides, $\mathrm{PCBs}$, and semi-volatile organic compounds in streambed sediment and fish on the Island of Oahu, Hawaii. Arch. Environ. Contam. Toxicol. 46 385-398.

BURGER AEB and MOOLMAN APM (2006) First phase of an endocrine research programme for South African water systems. Water Pract. Technol. 1 (2) 1-8.

BURGER AEC and NEL A (2008) Scoping Study to Determine the Potential Impact of Agricultural Chemical Substances (Pesticides) with Endocrine Disruptor Properties on Water Resources of South Africa. WRC Report No. 1774/1/08. Water Research Commission, Pretoria.

CAI Y, JIANG G and LIU J (2003) Solid-phase extraction of several phthalate esters from environmental water samples on column packed with polytetrafluoroethylene turning. Anal. Sci. 19 1491-1494

CAMPBELL CG, BORGLIN SE, GREEN FB, GRAYSON A, WOZEI E and STRINGFELLOW WT (2006) Biologically directed environmental monitoring, fate, and transport of estrogenic endocrine disrupting compounds in water: A review. Chemosphere $\mathbf{6 5}$ 1265-1280.

CASTILLO M, PUIG D and BARCELO D (1997) Determination of priority phenolic compounds in water and industrial effluents by polymeric liquid-solid extraction cartridges using automated sample preparation with extraction columns and liquid chromatography. Use of liquid-solid extraction cartridges for stabilization of phenols. J. Chromatography A 778 301-311.

CLOTFELTER ED, BELL AM and LEVERING KR (2004) The role of animal behaviour in the study of endocrine-disrupting chemicals. Anim. Behav. 68 665-676.

CLEDERA-CATRO M, SANTOS-MONTES A and IZQUIERDOHORNILLOS R (2005) Comparison of the performance of conventional microparticulates and monolithic reversed-phase columns for lipid chromatography separation of eleven pollutants phenols. J. Chromatography A 1087 57-63.

CLEDERA-CASTRO M, SANTOS-MONTES A and IZQUIERDOHORNILLOS R (2006) Method development and validation for phenol and nitrophenols in tap water by HPLC using a monolithic column. LCGC Europe 19 (7) URL: http://chromatographyonline. findanalytichem.com/lcgc/article/articleDetail.jsp?id=358051\&sk= $\&$ date $=\&$ page $\mathrm{ID}=8$. 
CORTAZAR E, BARTOLOME L, DELGADO A, ETXEBARRIA N, FERNÁNDEZ LA, USOBIAGA A and ZULOAGA O (2005) Optimisation of microwave-assisted extraction for the determination of nonylphenols and phthalate esters in sediment samples and comparison with pressurised solvent extraction. Anal. Chim. Acta 534 247-254.

CZAPLICKA M (2001) Determination of phenols and chlorophenols in bottom sediments. Suppl. 53 470-473.

DAWSON A (2000) Mechanism of endocrine disruption with particular reference to occurrence in avian wildlife: a review. Ecotoxicol. 9 395-399.

DE JAGER C, BORNMAN MS, VORSTER H, BURGER AEC and APPS PJA (1998) p-Nonylphenol in food packing material: an absorption study. Proc. Int. Symp. on Human Reproduction and Male Subfertility, 22-25 April, Genk, Belgium.

DIAZ-CRUZ MS, LOPEZ DE ALDA MJ and BARCELO D (2003) Environmental behavior and analysis of veterinary and human drugs in soils, sediments and sludge. Trends Anal. Chem. 22 340-350.

DUTY SM, ACKERMAN RM, CALAFAT AM and HAUSER R (2005) Personal care product use predicts urinary concentrations of some phthalate monoesters. Environ. Health Perspect. 113 (11) 1530-1535.

EBERLIN MN and CESAR DE SALIVA R (2008) Faster and simpler determination chlorophenols in water by fiber introduction mass spectrometry. Anal. Chim. Acta 620 97-102.

FALCONER IR, CHAPMAN HF, MOORE MR and RANMUTHUGALA G (2006) Endocrine-disrupting compounds: A review of their challenge to sustainable and safe water supply and water reuse. Environ. Toxicol. 21 181-191. DOI: 10.1002/tox.

FATOKI OS, BORNMAN M, RAVANDHALALA L, CHIMUKA L, GENTHE B and ADENIYI A (2010) Phthalate ester plasticizers in freshwater systems of Venda, South Africa and potential health effects. Water SA 36 (1) 117-126.

FATOKI OS and NOMA A (2002) Solid phase extraction method for selective determination of phthalate esters in the aquatic environment. Water, Air Soil Pollut. 140 85-98.

FERRAZ N, CARNEVIA D, NANDE G, ROSSOTTI M, MIGUEZ MN, LAST JA and GONZALEZ-SAPIENZA G (2007) Specific immunoassays for endocrine disruptor monitoring using recombinant antigens covered by degenerated primer PCR. Anal. Bioanal. Chem. 389 2195-2202.

FIAMEGOS YC, NANOS CG, PILIDIS GA and STALIKAS CD (2003) Phase-transfer catalytic determination of phenols as methylated derivatives by gas chromatography with flame ionization and mass-selective detection. J. Chromatography A 983 215-223.

GABRIEL LPF, CYRIS M, GIGER W and KOHLER HE (2007) Ipsosubstitution: A general biochemical and biodegradation mechanism to a cleave a-quartenary alkyl phenols and bisphenol A. Chem. Biodiversity 4 2123-2137.

GAME C, GAGNON MM, WEBB D and LIM R (2006) Endocrine disruption in male mosquitofish (Gambusia holbrooki) inhabiting wetlands in Western Australian. Ecotoxicol. 15 665-672.

GOVENDER S, JACOBS EP, BREDENKAMP MW and SWART P (2007) Affinity chromatography using biocompatible and reusable biotinylated membranes. J. Chromatogr. B 859 1-8.

HARTUNG R, LENOIR D, HENKELMANN B, SCHULTEHOSTEDE S and SCHRAMM K (2007) Reductive degradation of polychlorinated phenols by $\mathrm{Pd} / \mathrm{C}$-Formate: An ecoefficient remediation method for aqueous chlorinated phenols. Clean Soil Air Water 35 235-238.

HEBERER T and STAN H-J (1997) Detection of more than 50 substituted phenols as their t-butyldimethylsilyl derivatives using gas chromatography-mass spectrometry. Anal. Chim. Acta 341 21-34.

HECKER M and GIESY J P (2008) Novel trends in endocrine disrupting testing: The H295R steroidogenesis assay for identification of inducers and inhibitors of hormone production. Anal. Bioanal. Chem. 390 287-291.

HELALEH MIH, FUJI S and KORENAGA T (2001) Column Silylation method for determining endocrine disruptors from environmental water samples by solid phase micro-extraction. Talanta 54 1039-1047.
HILL SS, SHAWA BR and WUC AHB (2001) The clinical effects of plasticizers, antioxidants, and other contaminants in medical polyvinylchloride tubing during respiratory and non-respiratory exposure. Review. Clin. Chim. Acta 304 1-8.

HJELMBORG PS, GHISARI M and BONEFELD-JORGENSEN EC (2006) SPE-HPLC purification of endocrine disrupting compounds from human serum for assessment of xenoestrogenic activity. Anal. Bioanal. Chem. 385 875-887.

HOGBERG J, HANBERG A, BERGLUND M, SKERFVING S, REMBERGER M, CALAFAT AM, FILIPSSON AF, JANSSON B, JOHANSSON N, APPELGREN M and HAKANSSON H (2008) Phthalate diesters and their metabolites in human breast milk, blood or serum, and urine as biomarkers of exposure in vulnerable populations. Environ. Health Perspect. 116 (3) 334-339.

HUANG J, WANG H, JIN Q, LIU Y and WANG Y (2007) Removal of phenol from aqueous solution by adsorption onto OTMACmodified attapulgite. J. Environ. Manage. 84 229-236.

HUANG P-C, TIEN C-J, SUN Y-M, HSIEH C-Y and LEE C-C (2008) Occurrence of Phthalates in sediment and biota: Relationship to aquatic factors and the biota-sediment accumulation factor. Chemosphere 73 539-544.

JIAO B and CHENG CHK (2008) Disruption actions of bisphenol A and malachite green on growth hormone receptor gene expression and signal transduction in seabream. Fish Physiol. Biochem. 36 (2) 251-261. DOI: 10.1007/s10695-008-9227-0.

JUVANCZ Z, BARNA S, GYARMATHY D and KONOROT F (2008) Study of endocrine disrupting chemicals in the environment. Acta Polytech. Hungarica 5 (3) 49-58.

KAYALI N, TAMAYO FG and POLO-DIEZ LM (2006) Determination of dimethylhexyl phthalate in water by solid phase microextraction coupled to high performance liquid chromatography. Talanta 69 1095-1099.

KITANO T, KOYANAGI T, ADACHI R, SAKIMURA N, TAKAMUNE K and ABE SI (2006) Assesment of estrogenic chemicals using estrogen receptor $\alpha(E R \alpha)$ - and $E R \beta$-mediated reporter gene assay in fish. Mar. Biol. 149 49-55.

KRIJGSHEID KR and VAN DER GEN A (1986) Assessment of the impact of the emission of certain organochlorine compounds on the aquatic environment. Part 1: Monochlorophenols and 2,4-dichlorophenol. Chemosphere 15 (7) 825-860.

LASCOMBE I, BEFFA D, RUEGG U, TARRADELLAS J and WAHLI W (2000) Estrogenic activity assessment of the environmental chemicals using in vitro assays: identification of two new estrogenic compounds. Environ. Health Perspect. 108 621-629.

LATINI G, WITTASSEK M, DELVECHIO A, PRESTA G, DE FELICE C and ANGERER J (2009) Lactational exposure to phthalates in southern Italy. Environ. Int. 35 236-239.

LI D and PARK J (2001) Silyl derivatization of alkylphenols, chlorophenols and bisphenol A for simultaneous GC/MS determination. Anal. Chem. 73 3089-3095.

LI, X, ZHONG M, XUC S and SUN C (2006) Determination of phthalates in water samples using polyaniline-based solid-phase microextraction coupled with gas chromatography. J. Chromatogr. A. 1135 101-108.

LING W, GUI-BIN J, YA-QI C, BIN H., YA-WEI W and DA-ZHONG $S$ (2007) Cloud point extraction coupled with HPLC-UV for the determination of phthalate esters in environmental water samples. J. Environ. Sci. 19 874-878.

LIU R, ZHOU JL and WILDING A (2004) Simultaneous determination of endocrine disrupting phenolic compounds and steroid in water by solid phase extraction-gas chromatography-mass spectrometry. J. Chromatogr. A 1022 179-189.

LLOMPART M, LOURIDO M, LANDIN P, GARCIA-JARES C and CELA R (2002) Optimization of a derivatization - solid-phase microextraction method for the analysis of thirty phenolic pollutants in water samples. J. Chromatogr. A 963 137-148.

LUKS-BETLEJ K, POPP P, JANOSZKA B and PASCHKE H (2001) Solid-phase microextraction of phthalates from water. J. Chromatogr. A 938 93-101.

MAHOMED SI, VOYI KVV, ANECK-HAHN NH and DE JAGER C (2008) Oestrogenicity and chemical target analysis of water from small-sized industries in Pretoria, South Africa. Water SA 3 (3) 357-364.

Available on website http://www.wrc.org.za ISSN 0378-4738 (Print) = Water SA Vol. 36 No. 5 October 2010 ISSN 1816-7950 (On-line) $=$ Water SA Vol. 36 No. 5 October 2010 
MATTHIESSEN P (2000) Is endocrine disruption a significant ecological issue? Ecotoxicol. 9 21-24.

MAURICIO R, DINIZ M, PETROVIC M, AMARAL L, PERES I, BARCELO D and SANTANA F (2006) A characterization of selected endocrine disruptor compounds in a Portuguese wastewater treatment plant. Environ. Monit. Assess. 118 75-87.

McDOWELL DC and METCALFE CD (2001) Phthalate esters in sediments near a sewage treatment plant outflow in Hamilton Harbour, Ontario: SFE extraction and environmental distribution. J. Great Lakes Res. 27 3-9.

McKINLAY R, PLANT JA, BELL JNB and VOULVOULIS N (2008) Calculating human exposure to endocrine disrupting pesticides via agricultural and non-agricultural exposure routes. Sci. Total Environ. 398 1-12.

McNEELY RN, NEIMANIS VP and DWYER L (1979) Water Quality Source Book: A Guide to Water Quality Parameters. Water Quality Branch, Inland Waters Directorate, Environment Canada, Ottawa, Canada. 88 pp.

MODER M, BRAUN P, LANGE F, SCHRADER S and LORENZ W (2007) Determination of endocrine disrupting compounds and acidic drugs in water by coupling of derivatization, gas chromatograpgy and negative chemical ionization mass spectrometry. Clean Air Soil Water 35 (5) 444-451.

MOGHADAM M, TANGESTANINEJAD S, MIRKHANI V, MOHAMMADPOOR-BALTORK I, CHAHARDAHCHERIC S and TAVAKOLI Z (2008) Rapid and highly efficient trimethylsilylation of alcohols and phenols with hexamethyldisilazane (HDMS) catalyzed zirconyl triflate, $\left[\mathrm{ZrO}(\mathrm{OTf})_{2}\right]$. J. Organometal. Chem. 693 2041-2046.

MONTERO L, CONCRADI S, WEISS H and POPP P (2005) Determination of phenols in lake and ground water samples by stir bar sorptive extraction-thermal desorption-gas chromatographymass spectrometry. J. Chromatogr. A 1071 163-169.

MUSZKAT L, BIR L and RAUCHER D (1997) Identification of mixed O-phenyl alkyl phthalate esters in an agricultural land. Bull. Environ. Contam. Toxicol. 58 348-355.

NOPPE H, VERSLYCKE T DE, WULF E, VERHEYDEN K, MONTEYNE E, VAN CAETER P, JASSSEN CR and DE BRABANDER HF (2007) Occurrence of estrogens in the Scheldt estuary: a 2-year survey. Ecotoxicol. Environ. Saf. 6618.

OZKAYA M (2005) Chlorophenols in leachates originating from different landfills and aerobic composting plants. J. Hazard. Mater. 124 107-112.

PATANDIN S, LANTING CI, MULDER PG, BOERSMA ER, SAUER PJ and WEISGLAS-KUPERUS N (1999) Effects of environmental exposure to polychlorinated biphenyls and dioxins on cognitive abilities in Dutch children at 42 months of age. J. Pediatr. 134 (1) 33-41

PATROLECCO I, CAPRI S, DE ANGELIS S, POLESELLO S, VALSECCHI S (2004) Determination of endocrine disrupting chemicals in environmental solid matrices by extraction with a non-ionic surfactant (Tween 80). J. Chromatogr. A 1022 1-7.

PENG X, WANG Z, YANG C, CHENA F and MAIA B (2006) Simultaneous determination of endocrine-disrupting phenols and steroid estrogens in sediment by gas chromatography-mass spectrometry. J. Chromatogr. 1116 51-56.

PETROVIC M and BARCELO D (2000) Determination of anionic and nonionic surfactants, their degradation products, and endocrinedisrupting compounds in sewage sludge by liquid chromatography/ mass spectrometry. Anal. Chem. 72 4560-4567.

PETROVIC M, ELJARRAT E, LOPEZ DE ALDA MJ and BARCELO D (2001) Analysis and environmental levels of endocrine-disrupting compounds in fresh water sediments. Trends Anal. Chem. 20 (11) 637-648.

PETROVIC M, LACORTE S, VIANA P and BARCELO D (2002) Pressurized liquid extraction followed by liquid chromatographymass spectrometry for the determination of alkylphenolic compounds in river sediment. J. Chromatogr. A 959 15-23.

POLO M, LLOMPART M, GARCIA-JARES C and CELA R (2005) Multivariate optimization of a solid-phase microextraction method for the analysis of phthalate esters in environmental waters. J. Chromatogr. A 1072 63-72.
PSILLAKIS E, MANTAZAVINOS D and KALOGERAKIS N (2004) Monitoring the sonochemical degradation of phthalate esters in water using solid-phase microextraction. Chemosphere 54 849-857.

PUIG D and BARCELO D (1996) Determination of phenolic compounds in water and waste water. Trends Anal. Chem. 15 (8) 362-375.

RAJAPAKSE N, ONG D and KORTENKAMP A (2001) Defining the impact of weakly estrogenic chemicals on the action of steroidal estrogens. Toxicol. Sci. 60 296-304.

RIBEIRO A, NEVES MH, ALMEIDA MF, ALVES A and SANTOS L (2002) Direct determination of chlorophenols in landfill leachates by solid-phase micro-extraction-gas chromatography-mass spectrometry. J. Chromatogr. 975 267-274.

RICE C, BIRNABAUM LS, COGLIANO J, MAHAFFEY K, NEEDHAM L, ROGAN WJ and VOM SAAL FS (2003) Exposure assessment for endocrine disruptors: Some consideration in the design of studies. Environ. Health Perspect. 111 (3) 1683-1690.

ROMPA M, KREMER E and ZYGMUNT B (2003) Derivatisation in gas chromatographic determination of acidic herbicides in aqueous environmental samples. Anal. Bioanal. Chem. 377 590-599.

RUDEL RA and PEROVICH LJ (2009) Endocrine disrupting chemicals in indoor and outdoor air. Atmos. Environ. 43 170-181.

SANCHEZ-AVILA J, BONET J, VELASCO G and LACORTE S (2009) Determination and occurrence of phthalates, alkylphenols, bisphenol A, PBDEs, PCBs and PAHs in an industrial sewage grid discharging to a municipal wastewater treatment plant. Sci. Total Environ. 407 4157-6167.

SANTANA CM, FERRERA ZS, PADRON MET and RODRIGUEZ JJS (2009) Methodologies for the extraction of phenolic compounds from environmental samples: New approaches. Molecules 14 298-320.

SANTANA CM, FERRERA ZS and RODRIGUEZ JJS (2005) An environmentally friendly method for the extraction and determination of priority phenols in soils using micro-assisted micellar extraction. Anal. Bioanal. Chem. 382 125-133.

SARAJI M and BAKHSHI M (2005) Determination of phenols in water samples by single-drop microextraction followed by insyringe derivatization and gas chromatography-mass spectrometric detection. J. Chromatogr. A 1098 30-36.

SATHYANARAYANA S (2008) Phthalates and children's health. Curr. Probl. Pediatr. Adolesc. Health Care 38 34-49.

SCHMIDT K, STEINBERG CEW and STAAKS GBO and PFUGMACHER S (2005) Influence of a zenobiotic mixture (PCB \& TBT) compared to single substances on swimming behavior or reproduction Daphnia magna. Acta Hydrochim. Hydrobiol. 33 (4) 287-300

SCHMIDT-BAUMLER, HEBERER T and STAN HJ (1999) Occurrence and distribution of organic contaminants in the aquatic system in Berlin part II: Substituted phenols in Berlin surface water. Acta Hydrochim. Hydrobiol. 27 (3) 143-149.

SCHUMMER C, DELHOMME O, APPENZELLER BMR, WENNIG $R$ and MILLET M (2009) comparison of MTBSFA and BSTFA in derivation reaction of polar compounds prior to GC/MS analysis. Talanta 77 1473-1482.

SECKIN E, FROMME H and VOLEKL W (2009) Determination of total and free mono-butyl phthalate in human urine samples after medication of di-n-butyl phthalate containing capsule. Toxicol. Letters 188 33-37.

SEGNER H (2005) Developmental, reproductive, and demographic alterations in aquatic wildlife: Establishing causality between exposure to endocrine-active compounds (EACs) and effects. Acta Hydrochem. Hydrobiol. 33 (1) 17-26.

SHA Y, XIA X, YANG Z and HUANG GH (2007) Distribution of PAEs in the middle and lower reaches of the Yellow River, China. Environ. Monit. Assess. 124 277-287.

SHARPE R and SKAKKEBEAK NE (1993) Are estrogens involved in falling sperm counts and disorders of the male reproductive tract? Lancet 341 1259-1266.

SHIN J, MOON HJ, KANG H, KIM TS, LEE SJ, ALIN JY, BAE H, JEUNG EB and HAN SY (2007) OECD validation of the rodent Hershberger assay using three reference chemicals 17 $\alpha$-methyltestorone, procymidore and PP-DDE. Arch. Toxicol. 81 309-318. 
SOLOMON GM and SCHETTER T (2000) Endocrine disruption and potential health implications. Environ. Health 163 (11) 1471-1476.

SULIMAN FEO, AL-KINDI SS, AL-KINDY SMZ and AL-LAWATI HAJ (2006) Analysis of phenols in water by high-performance liquid chromatography using coumarin-6-sulfonyl chloride as a fluorogenic precolumn label. J. Chromatogr. A 1101 179-184.

SUN, WL, NI JR and LIU TT (2006) Effect of sediment humic substances on sorption of selected endocrine disruptors. Water, Air Soil Pollut. 6 583-591.

SUMPTER JP (2005) Endocrine disruptor in the aquatic environment: A review. Acta Hydrochem. Hydrobiol. 33 (1) 9-16.

SWAN SH (2008) Environmental phthalate exposure in relation to reproductive outcomes and other health endpoints in humans. Environ. Res., doi:10.1016/j.envres.2008.08.007.

SOTO AM, SONNENSCHEIN C, CHUNG KL, FERNANDEZ MF, OLEA N, OLEA-SERRANO MF (1995) The E-Screen assay as a tool to identify estrogens, an update on estrogenic environmental pollutants. Environ. Health Perspect. 103 (7) 113-122.

TERNES T, ANDERSEN H, GILBERG D and BONERZ M (2002) Determination of estrogens in sludge and sediments by liquid extraction and GC/MS/MS. Anal Chem. 74 (14) 3498-3504.

TONIOLO R, PIZZARIELLO A, SUSMEL S, DOSSI N, DOHERTY AP and BONTEMPELLI G (2007) An anionic-liquid based probe for sequential preconcentration from headspace and direct voltammetric detection of phenols in wastewater. Electrolysis 19 (19-20) 2141-2148.

TRENHOLM RA, VANDERFORD BJ, HOLADY JC, REXING DJ and SNYDER SA (2006) Broad range analysis of endocrine disruptors and pharmaceuticals using gas chromatography and liquid chromatography tandem mass spectrometry. Chemosphere 65 1990-1998.

TOPPARI J, LARSEN JC, CHRISTIANSEN P, GIWERCMAN A, GRANDJEAN P, GUILETTE LJ, JEGOU B, JENSEN TK, JOUANNET P, KEIDING N, LEFFERS H, MCLACHLAN JA, MEYER O, MULLER J, RAJPERT-DE MEYTS E, SCHEIKE T, SHARPE R, SUMPTER J and SKAKKEBAEK NE (1996) Male reproductive health and environmental xenoestrogens. Environ. Health Persp. 104 (4) 741-803.

US EPA (UNITED STATE ENVIRONMENTAL PROTECTION AGENCY) (1992) Guidelines for exposure assessment. EPA/600/Z-92/001. Risk assessment forum, US EPA, Washington, DC.

VAN DEN BERG M, SANDERSON T, KURIHARA N and KATAYAMA (2003) Role of metabolism in the endocrine-disrupting effects of chemicals in aquatic and terrestrial systems. Pure Appl. Chem. 75 (11-12) 1917-1932.

VAN WYK JH, POOL EJ and LESLIE AJ (2008) The effects of antiandrogenic and estrogenic disrupting cominants on breeding gland (nuptial pad) morphology, plasma testosterone levels, and plasma vitellogenin in male Xenopus laevis (African clawed frog) Arch. Environ. Contam. Toxicol. 44 247-256.

VAN WYK JH and PIETERSE GM (2003) A histo-morphological study of the testis of sharptooth catfish (Clarias gariepinus) as reference for future toxicological assessments. J. Appl. Ichthyol. 24 415-422.

VOGEL JM (2004) Tunnel vision: The regulation of endocrine disruptors. Pol. Sci. 37 277-303.

WANG L, CAI YQ, HE B, YUAN CG, SHEN DZ, SHAO J and JIANG GB (2006) Determination of estrogens in water by HPLC-UV using cloud point extraction. Talanta 70 47-51.

WELCH RM, LEVIN W and CONNEY AH (1969) Estrogenic properties of DDT and its analogs. Toxicol. Appl. Pharmacol. 14 358-367.

WHO (WORLD HEALTH ORGANIZATION) (2003) State of the Art Report: Health risks in aquifer recharge using reclaimed water. SDE/WSH/03.08. WHO, Geneva.

XUE N and XU X (2006) Composition, distribution and characterization of suspected endocrine-disrupting pesticides in Beying GuanTing Reservoir (GTR). Arch Environ. Contam. Toxicol. 50 463-473.

YING G-G, KOOKANA R, and WAITE TD (2004) Endocrine disrupting chemicals and pharmaceuticals and personal products (PPCPs) in reclaimed water in Australia. Australia Water Conservation and Research Programme Report.

YUAN SY, LIU C, LIAO CS and CHANG BV (2002) Occurrence and microbial degradation of phthalate esters in Taiwan river sediments. Chemosphere 49 1295-1299.

YUAN B, LI Z and GRAHAM N (2008) Aqueous oxidation of dimethyl phthalate in a $\mathrm{Fe}(\mathrm{VI})-\mathrm{TiO}_{2}-\mathrm{UV}$ reaction system. Water Res. 42 1413-1420.

ZALA SM and PENN D J (2004) Abnormal behaviours induced by chemical pollution: a review of the evidence and new challenges. Anim. Behav. 68 649-664.

ZENG F, CUI K, XIE Z, WU L, LUO D, CHEN L, LIN Y, LIU M and SUN G (2009) Distribution of phthalate esters in urban soils of subtropical city, Guangzhou, China. J. Hazard. Mater. 164 1171-1178.

ZHAO J, YING G, WANG L, YANG J, YANG X, YANG L and LI X (2009) Determination of phenolic endocrine disrupting chemicals and acidic pharmaceuticals in surface water of the Pearl Rivers in south China by gas chromatography-negative chemical ionizationmass spectrometry. Sci. Total Environ. 407 962-974.

ZHOU F, LI X and ZENG Z (2005) Determination of phenolic compounds in wastewater samples using a novel fiber by solid-phase microextraction coupled to gas chromatography. Anal. Chim. Acta 538 63-70. 